\title{
Optimisation of a parallel ocean general circulation model
}

\author{
M. I. Beare, D. P. Stevens \\ School of Mathematics, University of East Anglia, Norwich, NR4 7TJ, UK
}

Received: 6 January 1997 / Revised: 1 April 1997 / Accepted: 4 April 1997

\begin{abstract}
This paper presents the development of a general-purpose parallel ocean circulation model, for use on a wide range of computer platforms, from traditional scalar machines to workstation clusters and massively parallel processors. Parallelism is provided, as a modular option, via high-level message-passing routines, thus hiding the technical intricacies from the user. An initial implementation highlights that the parallel efficiency of the model is adversely affected by a number of factors, for which optimisations are discussed and implemented. The resulting ocean code is portable and, in particular, allows science to be achieved on local workstations that could otherwise only be undertaken on state-of-the-art supercomputers.
\end{abstract}

\section{Introduction}

As computing technology advances into the age of massively parallel processors (MPPs), ocean models are required that can utilise these state-of-the-art highperformance computers if oceanographers are to continue doing useful research. This is because the study of the ocean and its effect on the climate requires the resolution of features with small spatial scales and long time-scales over large geographic regions. Thus there is a requirement for ever more powerful computers, with large memory. The installation of a national MPP computer in the UK emphasises this technological shift in computing today. The Cray T3D, with 512 processors and a peak performance of nearly $80 \mathrm{Gflop} / \mathrm{s}$ is one of the fastest computers in the world. Unfortunately, as one of only a handful of tightly coupled parallel processors of this size, time on the Cray T3D is limited.

Correspondence to: M. I. Beare
It is essential therefore, that processing time is not needlessly wasted for the purpose of developing and testing codes. A parallel platform is required that is cheap and readily accessible, so that parallel codes can be developed and tested before porting to MPPs, such as the Cray T3D. Such platforms are also required for research which, whilst not requiring state-of-the-art supercomputers, still require large computational resources.

Recent developments in micro-processor, networking and software technologies have made it possible to treat a cluster of workstations as a single concurrent computer. A number of software packages have evolved that allow each connected computer to act as a processor in what is effectively a distributed-memory multi-processor machine, thus providing a platform on which to run parallel code. One popular package in use today is PVM (parallel virtual machine, Geist et al., 1993), which provides the programmer with a simple set of library routines to handle the communication between processors and their synchronisation. Given that many institutions already possess a number of workstations, which are often networked together, and that PVM is a portable public-domain package, the cost of setting up such an environment is just a few man-hours.

Within this paper we will describe the initial implementation of a parallel version of a general-purpose ocean circulation model. When run on a cluster of workstations it is seen that a number of bottlenecks contribute to a poor parallel efficiency. Techniques which consider interprocessor communications, load balancing and the model physics are then discussed and implemented to improve the performance of the code. The techniques employed tend to be relatively straightforward and aim to avoid over-complicating the code with the technical aspects of parallelism. Despite their simplicity, we show that these optimisations give rise to a significant increase in performance, such that the resulting model can be successfully and usefully run in parallel on a cluster of workstations, as well as on massively parallel processors. 
Throughout the paper, the workstation cluster used consists of sixteen DEC Alpha AXP 3000/300s, each with $32 \mathrm{Mb}$ of memory. This provides an environment that is homogeneous, although it should be noted that the model may also run in a heterogeneous environment. The cluster is configured to provide a general highperformance facility (Beare et al., 1997) and offers a dual networking ring, of Ethernet or FDDI (fibre distributed data interface) for parallel applications.

\section{The parallel model}

Many of the ocean models run today originate from the three-dimensional finite-difference model, based on the primitive equations of motion, described by Bryan (1969). His pioneering work has withstood the test of time and the only major changes were to optimise the code for running on vector computers and to include additional physics through new parameterisations (Semtner, 1974; Cox, 1984).

More recently the modular ocean model (MOM) code, as described by Pacanowski et al. (1991) and Pacanowski (1995), has been developed as a flexible research tool for oceanographers. MOM is modularised, using C-language pre-processor directives, allowing various physical options to be included or excluded as required. The MOM code has also been adapted for array processors, with a reduced set of options, by Webb (1996) and subsequently named MOMA (modular ocean model - array processor version). Although not strictly a parallel code, MOMA has been arranged in a way that allows the arrays to be vectorised in the vertical and decomposed in the horizontal. With a view to running fine-resolution global models on parallel processors, the rigid lid constraint, as used by Bryan, is removed in MOMA and a free surface is allowed (Killworth et al., 1991). It is the MOMA code upon which the parallel model developed in this paper is based.

The surface of the model ocean is assumed to be split into a two-dimensional horizontal grid. Each grid box is used to define a volume of water that extends from the surface to the ocean floor, organised into a number of vertical (depth) levels. The main prognostic variables required by the model are the baroclinic velocities and tracers (potential temperature and salinity), and the barotropic velocities and associated free-surface height field. The baroclinic variables describe the depth-dependent response of the ocean model and the barotropic variables describe the depth-averaged response. All other variables (such as density and vertical velocity) can be diagnosed from the prognostic variables and therefore the dynamic state of the ocean is described. The model variables are discretised using an Arakawa B-grid (Mesinger and Arakawa, 1976), so that the velocities are offset from the free-surface height and tracers. A finite-difference representation of the primitive equations is used to predict new values for these variables, thus allowing the model to be stepped forward in time using a series of discrete timesteps. Due to the fast speed of the external gravity waves, which must be considered when using the free-surface approach, the barotropic and baroclinic components are timestepped separately, with approximately 50-100 barotropic timesteps to each baroclinic timestep.

The model domain used in this paper is that of a pseudo-global ocean extending from $70^{\circ} \mathrm{S}$ to $70^{\circ} \mathrm{N}$. The maximum size of the longitudinal and latitudinal dimensions ( $I_{\max }$ and $J_{\max }$, respectively) is then dependent upon the required horizontal grid resolution. The vertical extent of the model is defined by a number of depth levels, which vary in thickness from about 20 to $500 \mathrm{~m}$. Levels below the ocean floor are masked and thus the topography of the ocean floor is described.

The basic mathematical model contains first- and second-order derivatives, so the finite-difference scheme requires gradients to be determined from values stored in neighbouring grid boxes. Problems occur when stepping forward variables at the perimeter of the model domain, using out-of-bounds array subscripts. The model arrays are therefore over-dimensioned by one grid point in the horizontal extents, creating an additional outer halo. In the case of a global model the outer halo in the longitudinal direction allows cyclic boundary conditions to be applied, where $(i=1) \equiv\left(i=I_{\max }-1\right)$ and $\left(i=I_{\max }\right) \equiv(i=2)$. In the latitudinal direction closed boundary conditions are applied to the northern and southern boundaries. The actual domain being modelled is therefore contained within the horizontal extents $\left(2: I_{\max }-1,2: j_{\max }-1\right)$.

To parallelise the ocean code a regular two-dimensional geometric data decomposition is applied to the model domain with respect to the horizontal plane. Each sub-domain is allocated to a processor, which is then responsible for storing and predicting the dynamic state of the ocean within that sub-domain. At the boundaries of these sub-domains, a similar problem to that found in the sequential version arises, with the possibility of outof-bounds array subscripts being used to reference adjacent grid points. Although these referenced grid points may exist in the context of the full model domain, they do not exist in terms of the local sub-domains. For calculations to be performed at these boundaries the processors require grid-point information that has been calculated on adjacent processors. This is achieved by passing the data from one processor to another, using high-level message passing routines, within the PVM environment. Figure 1 illustrates the message passing that is required between adjacent processors and how an additional halo is used to store overlapping grid points.

The concept of interprocessor communication, in our ocean model, is very similar to that of applying cyclic boundary conditions. Both schemes effectively copy data from one location to another to prevent out-ofbounds array referencing and both require an additional outer halo to do so. By taking this into account, parallelism can be easily implemented (at the top level) by replacing the cyclic boundary conditions with calls to the message passing routines. By enclosing the two respective sets of code in C-language pre-processor compiler directives, parallelism is offered as an option, 


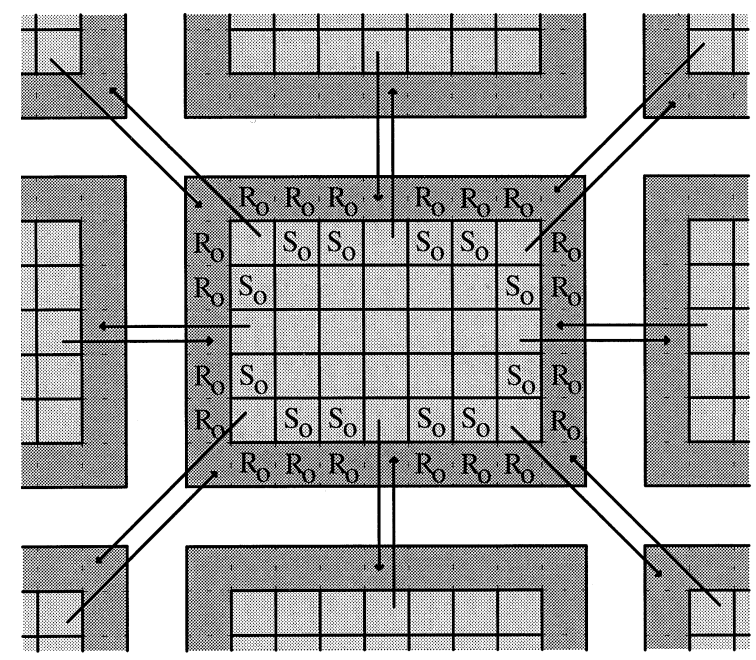

Fig. 1. To accommodate the exchange of boundary data, for a regular two-dimensional decomposition, an additional outer halo, $R_{0}$, is declared to receive the values that have been calculated and sent from the boundary halo, $S_{0}$, of adjacent sub domains

thus maintaining a modular structure to the code. A general overview of the code, with parallelism as a module option, is as follows:

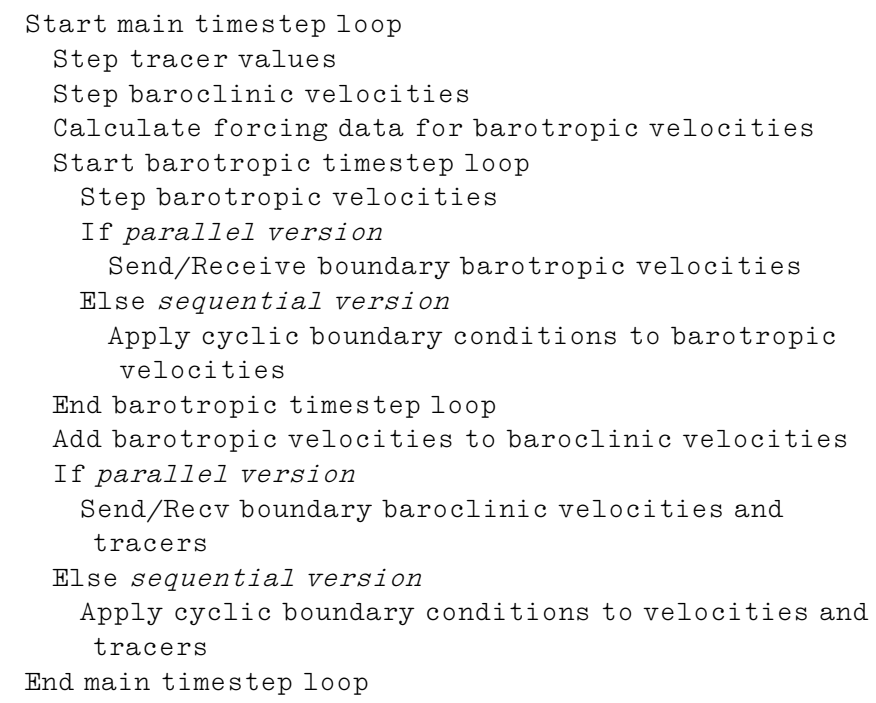

The following extract of FORTRAN 77 code highlights how the C-language pre-processor directives provide parallelism as an option, for the calculation of the baroclinic velocities. The subroutine clinic is called to calculate the new values for the velocity arrays, $u$ and $v$, at time $n p$ for a volume of depth, $k$, at each $i, j$ grid point. On completion of this process, either the highlevel message-passing routines are called, or cyclic boundary conditions are applied, depending on which running mode has been opted for. (It should be noted that it would be more efficient to have the $i, j$ loops inside the subroutine clinic, but the original sequential code did not and so we have left it unaltered, as it has no bearing on the parallel optimisations).

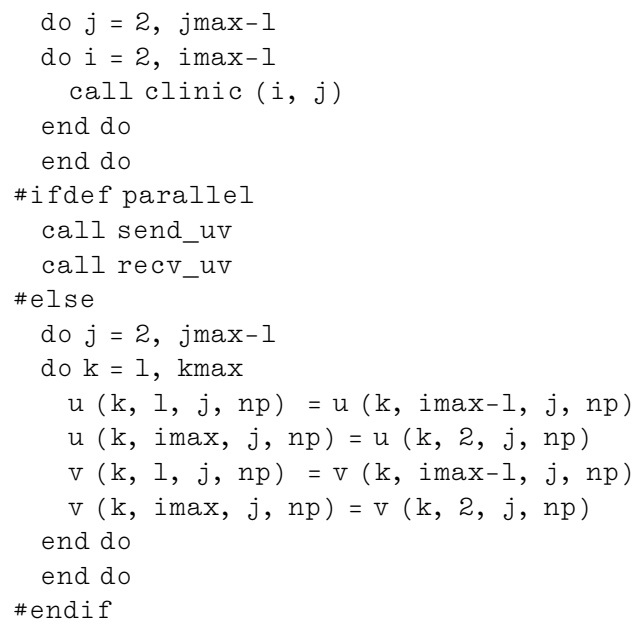

\section{Initial parallel implementation}

For an initial evaluation of the performance of the code the global model was defined with a two-degree horizontal resolution and 32 vertical depth levels. The model grid size is thus $32 \times 180 \times 69$, which leads to a moderate memory requirement of $20 \mathrm{Mb}$. The model was run for one model day on one to sixteen workstations, with a timestep of $30 \mathrm{~min}$. These runs were repeated over both interconnect systems (Ethernet and FDDI) and the results are plotted, in Fig. 2, alongside a run on a hypothetical, infinitely fast, interconnect system (achieved by doing no message passing, thus producing incorrect physics, but useful for timing purposes). It should be noted that for any number of

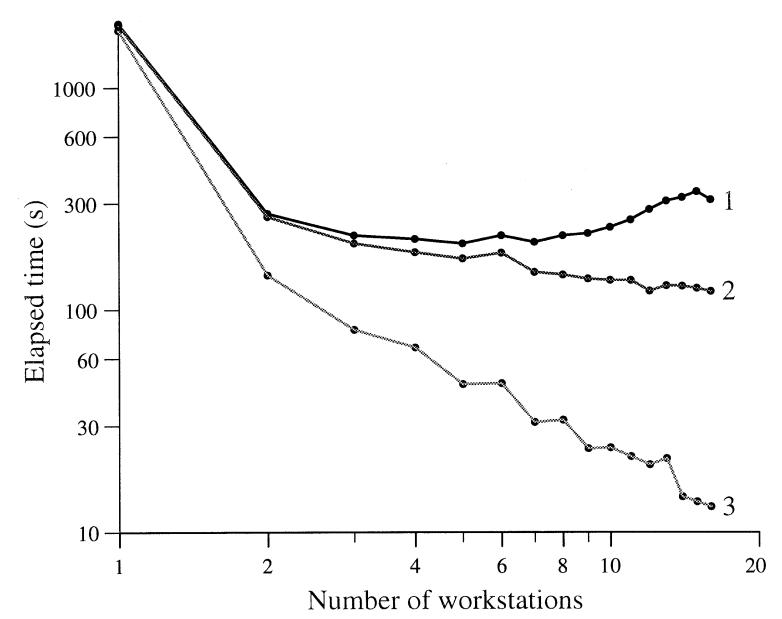

\begin{tabular}{|l|c|c|c|c|c|}
\hline & \multicolumn{5}{|c|}{ Number of workstations } \\
\cline { 2 - 6 } & 1 & 2 & 4 & 8 & 16 \\
\hline (1) Ethernet & 1950.7 & 270.0 & 207.4 & 215.6 & 312.4 \\
(2) FDDI & 1920.2 & 260.9 & 180.2 & 143.5 & 121.0 \\
(3) Ideal & 1822.5 & 142.7 & 67.5 & 31.9 & 13.0 \\
\hline
\end{tabular}

Fig. 2. The elapsed time required to model one day of a two-degree global model, with 32 levels, is plotted for runs using 1 to 16 workstations connected via (1) Ethernet, (2) FDDI and (3) a hypothetical, infinitely fast interconnect. For clarity, the table highlights a selection of the times plotted 
workstations the model domain can be decomposed in several ways, and different decompositions will often lead to different performance times. For example, with six processors the domain can be sub-divided as $6 \times 1$, $3 \times 2,2 \times 3$ or $1 \times 6$. So as not to complicate the graph only the best time recorded for a given number of workstations is plotted.

If the model were truly parallel efficient we would expect the performance to increase linearly with the number of workstations, such that two workstations would complete the run in the half the time taken by one workstation, and so on. This is clearly not the case with our model. To begin with the performance of the model does improve, but as more workstations are used the gain in performance decreases. In fact, when the interconnect is Ethernet, the performance becomes worse when more than five workstations are utilised.

The parallel efficiency on $P$ processors, $P E(P)$, relative to $P_{0}$ processors, is given by:

$P E(P)=\frac{E T\left(P_{0}\right)}{E T(P)} * \frac{P_{0}}{P} * 100$,

where $E T(P)$ is the elapsed time on $P$ processors. For the FDDI run, assuming $P_{0}=1$, we find that $P E(16)=99 \%$, which could be conceived as being very good. However, the model shows an initial super-linear speed-up, such that $P E(2)=368 \%$. This is due to the model being memory bound, when run on a single workstation, leading to a significant overhead when data is paged in and out of virtual memory. By running in parallel on two workstations, the memory requirements of the model are almost halved and (for this model resolution) the memory paging is eliminated. To remove this effect from our calculation of parallel efficiency, we choose $P_{0}=2$, which gives $P E(16)=27 \%$. Considering that lack of memory is often a prominent limitation when running large ocean models, it can therefore, in some cases, be advantageous to run the model on just two or three workstations. It is clear however, that if more workstations are to be used effectively, then the inefficiencies associated with the parallelism must be identified and resolved.

Further tests of the model determine three main areas of concern. The first of these is due to the calculation of the free-surface components (the barotropic mode). Compared to the rest of the numerical scheme, the amount of computation required to proceed one timestep is relatively small. However, as explained earlier, the timestep required to solve the free-surface variables must be much smaller than that for the baroclinic velocities or tracers. Hence more timesteps are required in order to step the free-surface forward the same amount of time as a single timestep would for the baroclinic mode. With each of these smaller timesteps there is a need to communicate newly solved values and so a comparatively large amount of time is consumed by communication rather than computation.

The second is due to the positioning of the message passing within the code. Having it coded neatly with the application of cyclic boundary conditions does make it easy to read and understand, but at the same time it is particularly inefficient. By exchanging data at the end of each timestep, after all the calculation has been completed, the processor is left with no alternative but to send its data and then sit idle, waiting for the complementary data to arrive from its neighbours.

The third problem is not so much to do with communications, but due to the regular domain decomposition used. Ocean models are made up of both ocean and land points, but since the model is only concerned with ocean points, the land points are masked and have no computation associated with them. Hence, although the model grid is split into evenly sized sub-domains, these domains do not necessarily consist of an equal number of ocean points and so processors will not always have equal amounts of work. This explains the irregular (saw-tooth-like) speed-up of the ideal case, which is not affected by communication overheads.

Discrepencies in times when running on a single processor are due to the model not checking whether or not it is possible to simply apply cyclic boundary conditions, instead of passing messages to itself. As will be seen later, this check is not essential, since in general, when running on a single workstation, the model will be run in sequential mode.

\section{Model optimisations}

To improve the parallel efficiency of the model code, each of the problem areas highlighted in the previous section will be looked at and the merits of implemented solutions are discussed. It should be remembered that these solutions aim to preserve the code's modularity and scalability, which are desirable features of the model that should not be overlooked in the search for efficiency.

\subsection{Low computation-communication ratio in the free surface}

The free-surface component of the model computes depth-averaged quantities which are stored in a twodimensional horizontal array of longitudinal and latitudinal grid points. Compared to the baroclinic mode there is a reduced number of variables that require solutions and a reduced number of boundary grid points that need to be shared with neighbouring processors. Unfortunately, the number of messages is not reduced and so the latency associated with the message passing is unchanged. Indeed an expensive overhead of PVM, on workstation clusters, is its high latency (Lewis and Cline, 1993) and in comparison to the smaller computation time, the communications become dominant in the time required to compute the free-surface component. The situation worsens as the number of processors increases and sub-domains get smaller.

The overhead due to the message passing within the barotropic mode can be clearly seen in Fig. 3, which depicts the results of four sets of runs: when just the baroclinic component is run (with and without message passing) and when just the barotropic component is run 


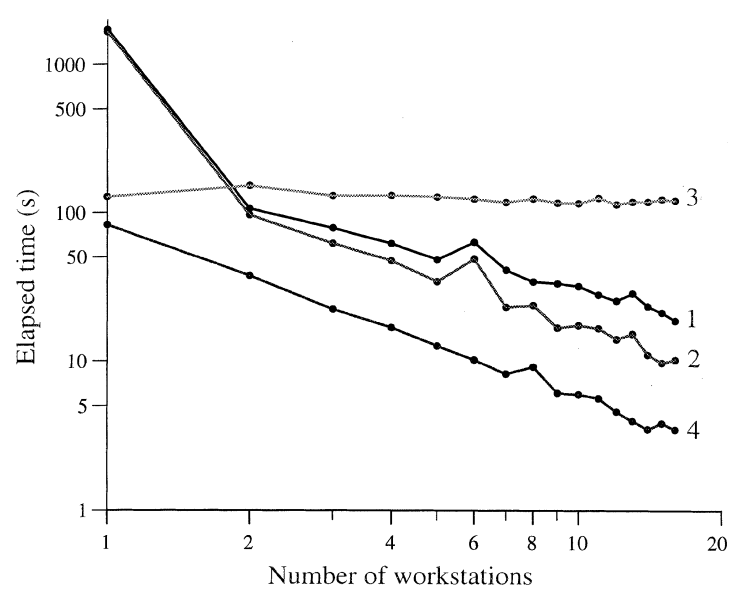

\begin{tabular}{|l|c|c|c|c|c|}
\hline & \multicolumn{5}{|c|}{ Number of workstations } \\
\cline { 2 - 6 } & 1 & 2 & 4 & 8 & 16 \\
\hline (1) B/C: with comms & 1723.7 & 106.5 & 62.0 & 34.2 & 18.7 \\
(2) B/C: no comms & 1655.8 & 96.5 & 47.3 & 23.7 & 10.2 \\
(3) B/T: with comms & 128.4 & 152.4 & 131.3 & 126.0 & 122.3 \\
(4) B/T: no comms & 82.5 & 37.4 & 16.8 & 9.2 & 3.5 \\
\hline
\end{tabular}

Fig. 3. Splitting the model into its baroclinic $(B / C)$ and barotropic $(\mathrm{B} / \mathrm{T})$ components and running these separately (over FDDI), with and without message passing, highlights the adverse communication overheads associated with the barotropic mode

(with and without message passing). Comparing the two runs in which the message passing is switched off highlights the fact that the barotropic component should compute much faster than the baroclinic part. Yet with message passing switched on, it is immediately clear that the communication associated with the free surface saturates the system, with a devastating affect on the overall performance of the model.

To resolve this problem a technique is adopted to minimise this communication, by trading it for a small amount of extra computation. By introducing further outer halos to the two-dimensional free-surface arrays, a greater amount of information can be exchanged between adjacent processors (as illustrated in Fig. 4). It is then possible to process a number of barotropic timesteps before having to stop and communicate. This approach is similar to that adopted by Sawdey et al. (1995), who define a number of extra fake zones, in order to reduce the frequency of communication.

In the initial implementation processors calculate new values for the different variables at each of the grid points within their domain, which is bounded by the halo $S_{0}$. For each timestep, an exchange of data between adjacent processors must occur. This message passing involves a processor sending values that have been calculated and stored in $S_{0}$, out to adjacent processors, which then receive and store these values in the halo $R_{0}$. The processors are then able to proceed with the next timestep.

Consider now the case of adding one extra halo, $R_{1}$, to the free-surface arrays, so that two halos can be sent and received for each data exchange. When message passing occurs, data from both the halos, $S_{0}$ and $S_{1}$, is packed and sent to adjacent processors, where it is received into the corresponding halos, $R_{0}$ and $R_{1}$. With this extra information a processor can calculate new values within the domain bounded by $R_{0}$ (as opposed to the domain bounded by $S_{0}$ ). Hence, on the next timestep, the values in the $R_{0}$ halo are already up to date and so no message passing is required in order to calculate the new values for the domain bounded by $S_{0}$. It is at the end of this second timestep that message passing will be required. Thus with one extra halo, message passing need only occur every other timestep and so the overall number of messages that are passed is halved, greatly reducing the relatively expensive latency times. Some extra computation is incurred, however, since values in the halo $R_{0}$ must be calculated, but this only need be done on the first of the two timesteps and with computation being so much faster than communication, this overhead is negligible.

The process of adding extra halos can be generalised to cope with any number of halos and Fig. 5 demonstrates the improved performance that can be achieved as the number of extra halos is increased (so as not to clutter the graph, only odd numbers of extra halos are plotted). The trade-off of communication for computation can give rise to a significantly better performance: almost $300 \%$ better for this example when using sixteen workstations connected via FDDI. There is however a limit to how far one can take this method and even with five extra halos the performance gain is beginning to tail off. Increasing the number of extra halos further eventually has a negative effect. Knowing how many extra halos can be usefully employed in this fashion is dependent on the number of workstations being used and the size and resolution of the model domain. One drawback of this optimisation method is however, that the code requires significant modifications to the freesurface routine, which add to its complexity.

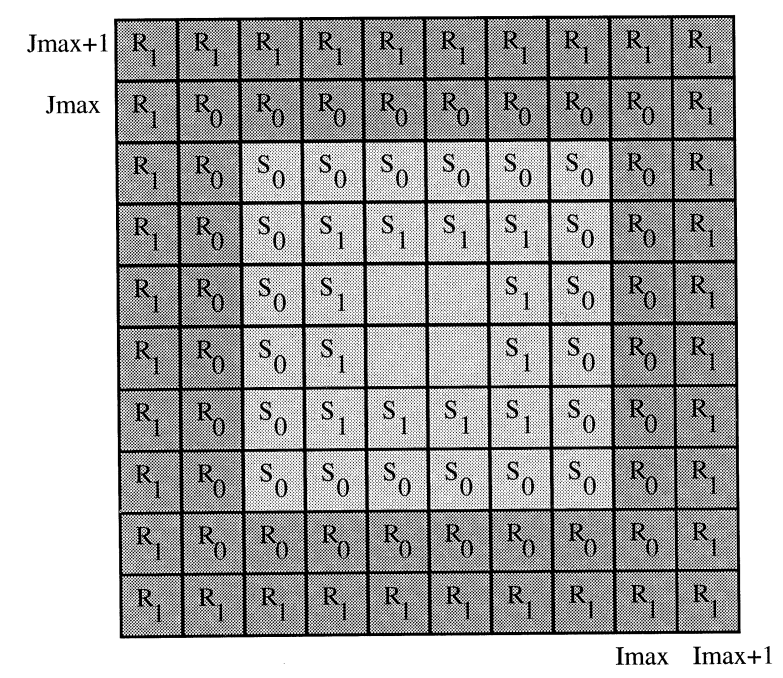

Fig. 4. To compute two barotropic timesteps, without requiring message passing, an extra outer halo, $R_{1}$, must be declared for receiving data from the inner halo, $S_{1}$. The halo, $R_{0}$, can then be stepped forward on the first of the two timesteps, so that the halo, $S_{0}$, can be calculated successfully in the second timestep 


\subsection{Reducing idle processor time}

The second inefficiency highlighted by the initial implementation is due to the idle time of processors waiting for communications to take place. Having solved all the finite-difference equations for a timestep, the dynamic variables at the boundaries are packed and sent to the appropriate neighbouring processors. Then, with no further calculations possible, the processors have no alternative but to sit idle, waiting for incoming messages to arrive, before they can continue processing. By recording the time spent in the receive routines, it is clear that idle time increases as the number of workstations increases, due to the additional messages travelling on the interconnect.

It is possible to minimise this idle time by rearranging the order in which the domain is parsed. By first calculating just those boundary values that need to be shared, it is possible to send the data and then, whilst this communication is taking place, calculate the values for the remaining inner grid points. If there is sufficient work within the inner domain to keep the processor busy, by the time all the new values have been calculated and it is time to receive the data sent from adjacent processors, the messages should have arrived and no idle time will be incurred.

To implement this method, the grid points at the boundary are identified by an ordered list of horizontal $(i, j)$ co-ordinate pairs and stored in an index array, as illustrated by Fig. 6. In detail, the following FORTRAN 77 code illustrates how this is implemented at the top level (again using the routine to predict baroclinic velocities as the example). The index array is outer and the number of outer-halo grid points that it references is nop.

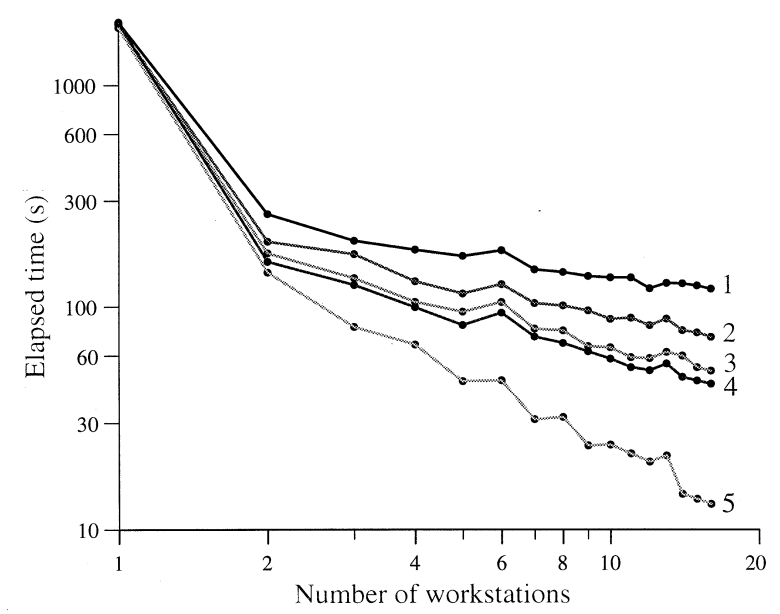

\begin{tabular}{|l|c|c|c|c|c|}
\hline \multirow{2}{*}{} & \multicolumn{5}{|c|}{ Number of workstations } \\
\cline { 2 - 6 } & 1 & 2 & 4 & 8 & 16 \\
\hline (1) Initial & 1920.2 & 260.9 & 180.2 & 143.5 & 121.0 \\
(2) $1 \mathrm{XH}$ & 1872.4 & 196.1 & 130.0 & 101.2 & 73.4 \\
(3) $3 \mathrm{XH}$ & 1892.5 & 174.0 & 105.0 & 78.3 & 51.7 \\
(4) $5 \mathrm{XH}$ & 1902.4 & 159.6 & 99.6 & 68.8 & 45.1 \\
(5) Ideal & 1822.5 & 142.7 & 67.5 & 31.9 & 13.0 \\
\hline
\end{tabular}

Fig. 5. Compared to (1) the initial implementation (when using FDDI), the performance of the code is significantly improved as the number of free-surface extra halos $(X H)$ is increased in (2), (3) and (4)

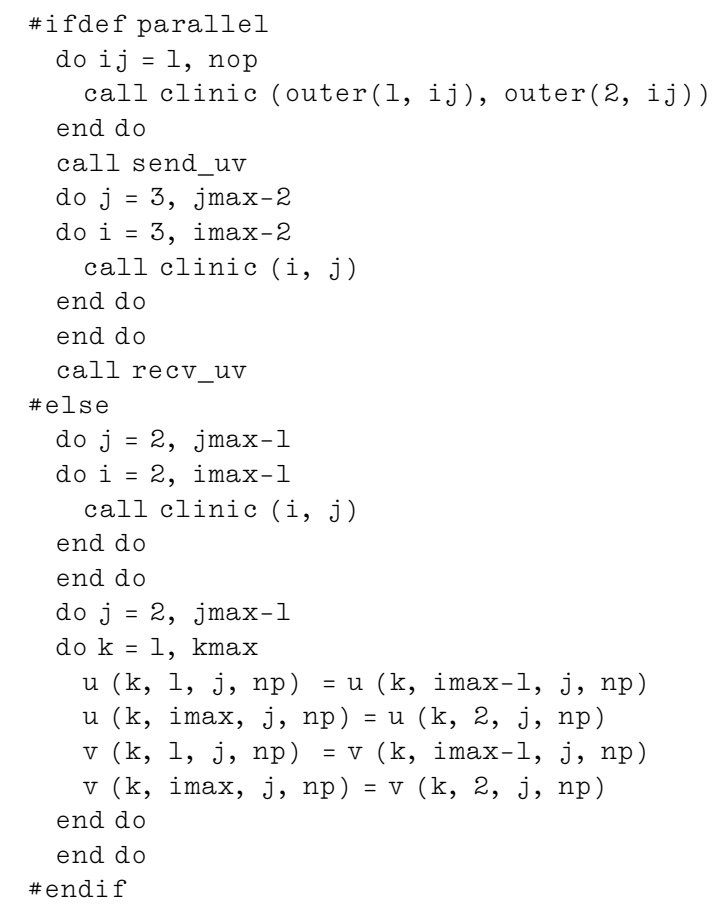

Figure 7 highlights the potential of this optimisation, with the time taken to complete a model day being more than halved, compared to the initial implementation. This is comparable to the performance increase gained by the previous free-surface optimisation, but is much simpler to implement and leads to a tidier code. A potential disadvantage of this optimisation is that it can lead to an inefficient utilisation of cache memory. This is evident when comparing the performance of the parallel codes when run on a single workstation. In the initial implementation the horizontal subscripts $(i, j)$ are parsed in the order in which the arrays are stored in memory, thus making efficient use of the cache. However, by solving the equations for the outer-halo grid points before the inner ones, this efficiency is compromised. Despite this, the benefits of reducing the idle time of each processor, when running on more than one processor, gives a significant improvement on the overall performance of the model. Also, as suggested earlier, when using a single processor the model will be run in

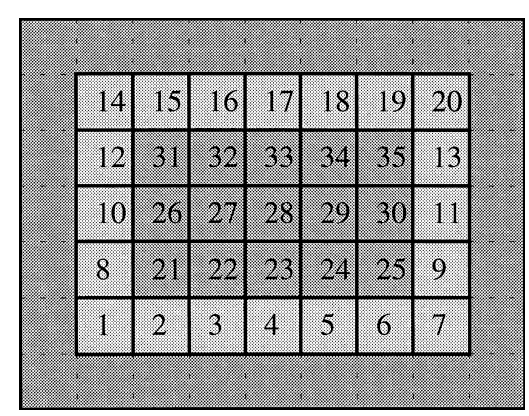

Fig. 6. Reordering the way in which the horizontal grid points are parsed, so that the boundary halo (numbered $1-20$ ) is completed first, allows the communication of this boundary data to be overlapped with the calculation of the remaining inner grid points (21-35) 
sequential mode and will therefore revert back to the original, more efficient, method of parsing the domain.

\subsection{Combining the optimisations}

As stated earlier, the technique used to reduce the communication overheads of the free-surface routine complicates the code to some extent and this would be complicated further if, in its general form, it was combined with the technique of overlapping communications with computation. A reduced implementation of the extra free-surface halo technique is therefore opted for. Instead of having a generalised algorithm that is able to cope with any number of extra halos, the number is fixed to be just one extra halo (i.e. two halos are transferred). This simplifies the implementation of the optimisation and the code remains comprehensible to the ocean modeller. Furthermore, other ocean physics that may subsequently be added to the code, might also require message passing routines to transfer two halos of data. Such options include the QUICK tracer advection scheme (Farrow and Stevens, 1995) and biharmonic mixing, which have finite-difference representations that span five grid points.

Figure 8 gives a summary of the performance of each of the discussed implementations when run on the workstation cluster connected by FDDI. It is evident that the final optimised code can give rise to a threetimes speed-up over the initial parallel implementation, completing one model day in $40.4 \mathrm{~s}$ compared to $121.0 \mathrm{~s}$, when using sixteen workstations.

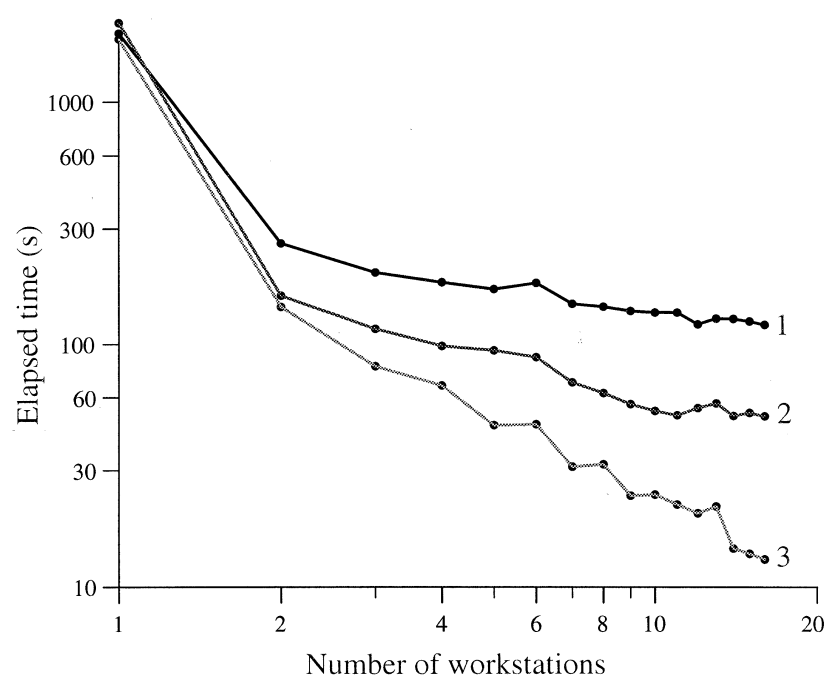

\begin{tabular}{|l|c|c|c|c|c|}
\hline & \multicolumn{5}{|c|}{ Number of workstations } \\
\cline { 2 - 6 } & 1 & 2 & 4 & 8 & 16 \\
\hline (1) Initial & 1920.2 & 260.9 & 180.2 & 143.5 & 121.0 \\
(2) Overlap & 2126.9 & 158.4 & 98.5 & 62.8 & 50.5 \\
(3) Ideal & 1822.5 & 142.7 & 67.5 & 31.9 & 13.0 \\
\hline
\end{tabular}

Fig. 7. Reducing idle processor time, by (2) overlapping computation with inter-processor communications, leads to an improved performance over (1) the initial implementation (for the 1-day model run, using FDDI)

\subsection{Load balancing}

The use of a regular domain decomposition, which allows for easier parallelisation and greater scalability and modularity, does not however, distribute equal amounts of work to each of the processors. As well as ocean points, the model may also consist of land points, which have no computation associated with them. Due to the uneven distribution of these land points within the domain, it is not inconceivable for some processors to end up with more work than others and a few processors may even be allocated no work at all.

To lessen this imbalance, one solution is to divide the domain into a greater number of sub-domains than there are processors (Sawdey et al., 1995). The objective is to end up with the same number of sub-domains, containing some work, as there are processors. Those sub-domains with no work can then be ignored when allocating sub-domains to processors, as illustrated in Fig. 9.

This approach forms an elegant solution that is easy to implement, requiring changes only to the initial processor allocation phase (which occurs at compilation time, when the machine configuration is chosen). The advantages are that hopefully no processors will remain idle and that the maximum size of the sub-domains is reduced, thus making the overall performance of the parallel model much better. Also, due to the smaller sized sub-domains, less memory is required per processor. It should be noted that the more processors that are available the more successful this approach will be and it is therefore more suitable when running on MPPs. Table 1 illustrates this technique for the two-degree model with varying decompositions when using 256 processors. For this model an ideal allocation for 256 processors would give a maximum of 1050 ocean points per processor. Although not ideal, this approach can greatly reduce the maximum amount of work per processor and reduce the memory requirements. In this case a $25 \%$ reduction is achieved when using a $20 \times 15$ decomposition.

To get an ideally balanced load would require an irregular two-dimensional decomposition, such as that implemented in the OCCAM (ocean circulation and climate advanced modelling) code and described by Gwilliam (1995). This may however give rise to other problems, not present in our parallel model. In particular, due to the static nature of FORTRAN 77 the arrays must be dimensioned identically for each processor, with scalar variables indicating the true extents of each sub-domain. Thus, if a horizontal decomposition results in a $12 \times 6$ and a $5 \times 14$ sub-domain, amongst others, then all arrays would have to be declared as at least $12 \times 14$. Thus the memory requirements do not necessarily scale well as the number of processors increase, which in turn could inhibit the degree of resolution of the model.

When using just a few processors, it may be beneficial to restrict decomposition to one dimension and use irregular-sized domains to achieve a more evenly balanced load, as used by Sadwey et al. (1995). However, 


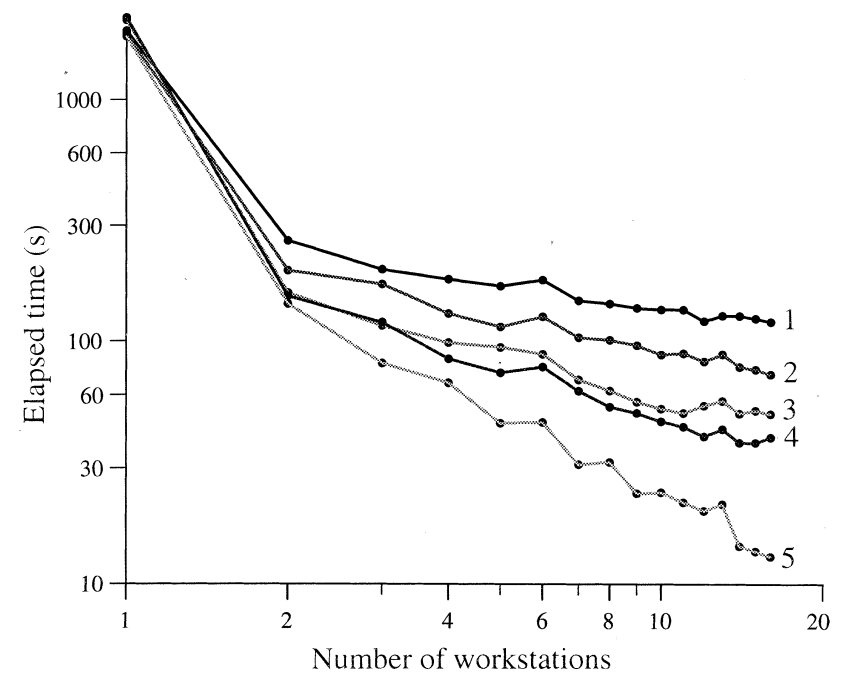

\begin{tabular}{|l|c|c|c|c|c|}
\hline \multirow{2}{*}{} & \multicolumn{5}{|c|}{ Number of workstations } \\
\cline { 2 - 6 } & 1 & 2 & 4 & 8 & 16 \\
\hline (1) Initial & 1920.2 & 260.9 & 180.2 & 143.5 & 121.0 \\
(2) I XH & 1872.4 & 196.1 & 130.0 & 101.2 & 73.4 \\
(3) Overlap & 2126.9 & 158.4 & 98.5 & 62.8 & 50.5 \\
(4) Combined & 2184.4 & 153.9 & 84.6 & 54.0 & 40.4 \\
(5) Ideal & 1822.5 & 142.7 & 67.5 & 31.9 & 13.0 \\
\hline
\end{tabular}

Fig. 8. Compared to (1) the initial implementation and the two separate optimisations, of (2) one extra free-surface halo and (3) overlapping communications with computation, it can be seen that by (4) combining the optimisations a notable increase in performance is again achieved

this approach also suffers from memory not scaling with numbers of processors, and care needs to be taken to ensure that memory paging is not reintroduced. When using workstation clusters, it is not necessarily true that all workstations will be of equal power, and another possible solution may be to simply match the subdomains with higher work loads to the higher-powered workstations.

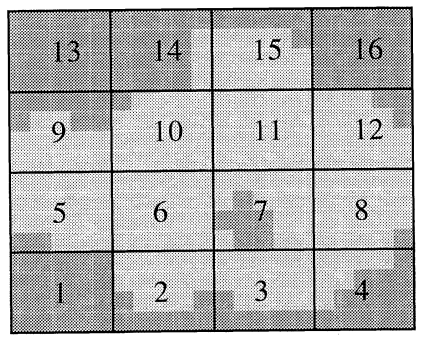

a

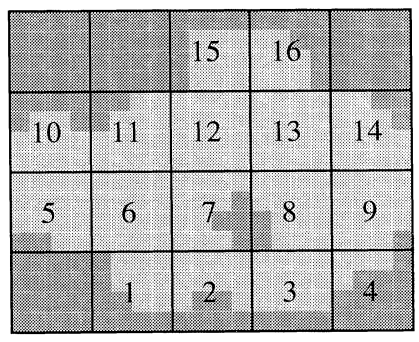

b
Fig. 9a,b. A regular two-dimensional decomposition of an ocean model, containing land (dark-shaded) and ocean (light-shaded) points, can result in some processors having no work (as highlighted in a by processors 1, 13 and 16). By sub-dividing the domain into a greater number of smaller sub-domains, those with no work can be ignored completely and a better utilisation of the sixteen available processors can be achieved (as highlighted in b)

\section{Model evaluation}

So far, the results discussed have been for the model running in parallel mode, even for single workstation runs. However, when using a single workstation, it is more efficient to run the model in sequential mode. Firstly, the code does not go through the motions of computing outer-halo values before inner grid values and so cache memory is better utilised. Secondly, the parallel version makes no attempt to identify the fact that it is running on a single workstation and therefore proceeds to do message passing to apply cyclic boundary conditions. For a true picture of speed-up it is necessary to compare the performance of the parallel model against that of the sequential one.

In the case of the model that has been used throughout this paper, the sequential version ran for one model day in a time of $1820 \mathrm{~s}$. This implies that the time of $40.4 \mathrm{~s}$ when run on sixteen workstations represents a speed-up of 45.0. It has, however, already been stated that memory paging occurs on a single workstation with $32 \mathrm{Mb}$ of memory. To gauge the relative speed-up if this paging were not a factor, the model was run on a $96 \mathrm{Mb}$ workstation. Here the sequential version ran in $259 \mathrm{~s}$, so the estimated scaled speed-up when using sixteen workstations is 6.4. For the code to be more effective over sixteen workstations, it is necessary to increase the size of the model. Running larger, finer-resolution ocean models is, however, one of the main rationales for parallelism.

The same pseudo-global model but with a one-degree horizontal resolution was declared and run for one model day, with a 45-min timestep. This time, the $96 \mathrm{Mb}$ workstation was also forced to page memory when running the sequential version and took $2173 \mathrm{~s}$ to complete one model day. On the sixteen $32 \mathrm{Mb}$ workstations, the time taken was just $76 \mathrm{~s}$, representing a speed-up of 28.5. Reducing the number of depth levels to 24 enables the model to fit into memory on the $96 \mathrm{Mb}$ workstation and a truer picture of speed-up can be seen. With a time of $617 \mathrm{~s}$ for the sequential version, a speedup of 10.9 is achieved by the sixteen workstations, which take $57 \mathrm{~s}$ to run. Thus it would take just under $6 \mathrm{~h}$ to model 1 year on this cluster.

Increasing the size of the model further, by defining a finer half-degree horizontal resolution (again with 24 levels), produces a model that just a few years ago, only the largest research groups could envisage running (Semtner and Chervin, 1992) on the fastest supercomputers of the day. On the cluster, this half-degree global

Table 1. 256 processors can be used more efficiently by subdividing the model into greater than 256 sub-domains and ignoring those domains with no associated work

\begin{tabular}{llll}
\hline $\begin{array}{l}\text { Processor } \\
\text { decomposition }\end{array}$ & $\begin{array}{l}\text { number of } \\
\text { sub-domains }\end{array}$ & $\begin{array}{l}\text { max. ocean pts. } \\
\text { per sub-domain }\end{array}$ & $\begin{array}{l}\text { sub-domains } \\
\text { with some work }\end{array}$ \\
\hline $16 \times 16$ & 256 & 1920 & 223 \\
$18 \times 17$ & 306 & 1600 & 256 \\
$20 \times 15$ & 300 & 1440 & 255 \\
\hline
\end{tabular}


model, with a 22.5-min timestep, takes just under $63 \mathrm{~h}$ to complete a 1 -year run.

Throughout these tests on the workstation cluster, a one-dimensional decomposition always proved more efficient than a two-dimensional decomposition. This, despite the fact that in general a one-dimensional decomposition actually requires more grid points to be communicated for a given number of processors. The reason for the one-dimensional decomposition being more efficient, as seen before, is due to message latency, not the amount of data being transferred. With a twodimensional decomposition processors can have up to eight neighbours that they need to communicate with, hence up to eight messages need to be created and sent each time any communication is required. With a onedimensional decomposition, there is a maximum of only two neighbours and so less messages need be sent.

When ported to the Cray-T3D, with its fast point-topoint interconnect, it was found that interprocessor communications were not as much of a bottleneck as on the workstations. A two-dimensional decomposition was therefore no less efficient than a one-dimensional one: in fact it offers better load-balancing prospects. The benefits of the load-balancing optimisation technique described in Sect. 4.4 can be seen when the preceding half-degree model is run on 256 processors. A $16 \times 16$ decomposition results in a time of $69.8 \mathrm{~s}$ for a 1-day model run, with 33 processors remaining idle, whereas when using a $20 \times 15$ decomposition the available processors are better utilised and the run takes only $60.6 \mathrm{~s}$ to complete. Hence a year-long run can be completed in just over $6 \mathrm{~h}$.

By maintaining the modularity of the code and providing parallelism as a high-level option, the model is not dissimilar in appearance to the original sequential code. It is therefore considered that this will provide ocean modellers with a familiar code that they can use easily, without having to concern themselves with the technical aspects of parallelism. Modifying and extending the code is relatively straightforward, with the addition and development of new physical options being implemented as usual in sequential mode. Once these have been proven correct, the high-level message passing routines can be called from the appropriate points (i.e. wherever cyclic boundary conditions are applied) and the parallel results can then be validated against the sequential results.

Although PVM aids portability, it is not the most efficient of message-passing systems and it is envisaged that at a later date the high-level routines could be rewritten for other portable environments, such as MPI (message passing interface forum, 1994), or include machine specific options, such as SHMEM on the Cray T3D.

With the code discussed in this paper it is possible to modify, test and run the ocean model on local workstations, before porting to national MPPs, on which resources are limited. Hence these resources can be used much more productively. Furthermore, groups without access to MPP systems are now able to run large models on readily accessible local workstations, which may be underutilised overnight.

Acknowledgements. We would like to acknowledge the work of David Webb at Southampton Oceanography Centre towards MOMA, upon which this parallel model has been based. We also wish to thank the Computing Centre at the University of East Anglia for their committment to providing a central service high performance parallel facility, based around their Alpha Farm. M. I. Beare was partially supported by NERC research grants GR3/8526 and GST/02/1012.

Topical Editor D. J. Webb thanks M. Ashworth and S. Maskell for their help in evaluating this paper.

\section{References}

Beare, M. I., A. P. Boswell, and K. L. Woods, Managing a central service cluster computing environment, AXIS: UCISA J. Acad. Comput. Inf. Syst., (in press), 1997.

Bryan, K., A numerical method for the study of the circulation of the world ocean, J. Comput. Phys., 4, 347-376, 1969.

Cox, M. D., A primitive equation, three-dimensional model of the ocean, GFDL Ocean Tech. Rep. 1, Geophysical Fluid Dynamics Laboratory/NOAA, Princeton Univ., Princeton, NJ, 1984.

Farrow, D. E., and D. P. Stevens, A new tracer advection scheme for Bryan and Cox type ocean global circulation models, $J$. Phys. Oceanogr., 25, 1731-1741, 1995.

Geist, A., A. Beguelin, J. Dongarra, R. Manchek, and V. Sunderam, PVM 3 users guide and reference manual, Oak Ridge National Laboratory, Oak Ridge, Tennessee, 1993.

Gwilliam, C. S., The OCCAM global ocean model, in Proc. Sixth ECMWF Worksh. on the use of Parallel Processing in Meteorology, Eds. G. Hoffman and N. Kreitz, World Scientific, London, pp. 446-454, 1995.

Killworth, P. D., D. Stainforth, D. J. Webb, and S. M. Paterson, The development of a free-surface Bryan-Cox-Semtner ocean model, J. Phys. Oceanogr., 21, 1333-1348, 1991.

Lewis, M., and R. E. Cline Jr., PVM communication performance in a switched FDDI heterogeneous distributed computing environment, Sandia National Labs, Tech. Rep., 1993.

Mesinger, F., and A. Arakawa, Numerical methods used in atmospheric models, GARP publications series, No.17, World Meteorological Organisation, Geneva, 1976.

Message Passing Interface Forum, MPI: A message passing interface standard, Univ. of Tennessee, Rep. CS-94-230, 1994.

Pacanowski, R. C., MOM2 documentation, user's guide and reference manual, GFDL Ocean Tech. Rep. 2, Geophysical Fluid Dynamics Laboratory/NOAA, Princeton Univ., Princeton, NJ, 1995.

Pacanowski, R. C., K. Dixon, and A. Rosati, The GFDL modular ocean model users guide: version 1.0, GFDL Ocean Tech. Rep. 2, Geophysical Fluid Dynamics Laboratory/NOAA, Princeton Univ., Princeton, NJ, 1991.

Sawdey, A., M. O'Keefe, R. Bleck, and R. W. Numrich, The design, implementation and performance of a parallel ocean circulation model, Proc. Sixth ECMWF Worksh. on the use of Parallel Processing in Meteorology, Eds. G. Hoffman and N. Kreitz, World Scientific, London, pp. 523-548, 1995.

Semtner, A. J., A general circulation model for the world ocean, Tech. Rep. 9, Dept. of Meteorology, Univ. of California, Los Angeles, 1974.

Semtner, A. J., and R. M. Chervin, Ocean general circulation from a global eddy-resolving model, J. Geophys. Res., 97, 5493-5550, 1992.

Webb, D. J., An ocean model code for array processor Computers, Comput. Geosci., 22, 569-578, 1996. 